Tafel 154. Christian Peter Hansen, 1803-1879. Chronist von Sylt. / Erich Johannsen, 1862-1938. Heimatdichter von Sylt.

" 155. Reisehügel bei Braderup auf Sylt. Residenz des Zwergkönigs Finn. / Holstich oder Wullstich bei Kampen auf Sylt.

" 156. Verschworenenweg bei der Vogelkoje auf Amrum. / Osetal bei Wenningstedt auf Sylt.

" 157. Titelblatt der Chronik von Heimreich mit der Darstellung der beiden Hauptgötter der Friesen Fhoseta oder Fosta und Waeda oder Woeda.

" 158. Ausschnitt aus der Karte „Nordertheil vom Alt Nordt Frisslande bis an das Jahr 1240“ von Joh. Meyer, Husum 1649. / Balckstein im Wattenmeer nördlich von Dunsum auf Föhr.

„ 159. Fundamentsteine der 1634 untergegangenen Kirche von Morsum auf Alt-Nordstrand, im Wattenmeer zur Ebbezeit, von $O$ nach $W$ gesehen. / Friedhof bei Hallig Habel, vermutlich von vor 1362, zur Ebbezeit im Wattenmeer freiliegend.

" I6o. Friedhof der Kirchwarf von Gröde während einer Überflutung der Hallig bei Südweststurm am 20. 9. 1935. / Blick vom Kirchturm der St. Severin-Kirche von Keitum auf Sylt auf den Friedhof.

" 161. Kirche der Hallig Gréde von 1779. / St. Nicolai-Kirche in Boldixum auf Föhr. Anfang des 13. Jahrhunderts.

„ 162. St. Laurentii-Kirche bei Süderende auf Föhr. Anfang des I3. Jahrhunderts. / St. Johannis-Kirche von Nieblum auf Föhr. Ende des 12. Jahrhunderts.

„ I63. St. Martin-Kirche von Morsum auf Sylt. Ende des I 2. Jahrhunderts. / St. Martin-Kirche

" von Morsum auf Sylt. Südseite des Chores mit Fries und Bleidach von 1790.

164. St. Severin-Kirche von Keitum auf Sylt. Ende des 12. Jahrhunderts. / St. Severin-Kirche von Keitum auf Sylt. Apsis mit Fries und Fenster.

" 165. Grabstein des Kapităns Dirck Cramers, 1725-1769, in Nieblum auf Föhr. / Grabstein von Peter Melffsen, Anfang des I8. Jahrhunderts, Boldixum auf Föhr. / Grabstein des Müllers Hans Cristiansen, 1685-1771, in Nieblum auf Föhr.

166. Grabstein des Schiffers Oluf Jensen, $1672-1750$, in Nebel auf Amrum, Vorderseite und Rückseite.

„167. Sonnenuntergang auf Sylt.

\title{
VOM HERAUSGEBER BENUTZTE QUELLEN
}

Ahlborn, Knud, und Goebel, Ferdinand. Das Syltbuch. Kampen. (Mit Literaturangaben.)

Alnor, Karl. Uwe Jens Lornsen. Eine historisch-politische Skizze. Flensburg.

Ambrosius, Eduard Arnold. Kurze Nachrichten von der Insel Sylt. I. Auflage 1 792. Neuherausgegeben und mit Anmerkungen versehen von Herm. Schmidt, Westerland 1935.

Andresen, Ludwig. Kultur-Spuren im Watt bei der Hallig Langeneß-Nordmarsch. Föhrer Heimatbücher Nr. 22. Hamburg 1937.

Ball, Friedrich. Strandungen an der Küste von Sylt. Westerland 1930.

Baudissin, Graf Adelbert. Schleswig-Holstein meerumschlungen. Stuttgart 1865 . Blicke in die Zukunft der nordfriesischen Inseln und der Schleswigschen Festlandsküste. Schleswig 1867.

Behrmann, Walter. Über die niederdeutschen Seebücher des fünfzehnten und sechzehnten Jahrhunderts. Hamburg 1906.

Biernatzki, J. C. Die Hallig oder die Schiffbrüchigen auf dem Eiland in der Nordisee. Leipzig 1836.

Boehn, Max von. Puppen. München 1929.

Boeles, P. C. J. A. Friesland tot de elfde eeuw. Zyn oudste beschaving en geschiedenis. '\&-Gravenhage 1927.

Boetius, Mathias. „de cataclysmo Nordstrandico" oder des Matthias Boetius drei Bücher uber Denkwürdigkeiten von Sturmfluten, welche Nordstrand betroffen haben. Schleswig 1623. Ubersetzt von Dr. Schmidt-Petersen, Bredstedt, veröffentlicht im Jahrbuch des Nordfriesischen Vereins, Heft 193 I-1933.

Boetius, Steffen. Eheliches Güterrecht und Erbrecht auf Osterlandföhr und in Wyk vor Igoo. Leipzig 1928.

Boie, Karl. Schleswig-Holsteinische Siegel des Mittelalters. Neumünster 193 I. 
Boie, Margarete. Der Sylter Hahn. Stuttgart 1925 .

Waal - Waal. Das Leben eines Sylter Grönlandfahrers. 2. Auflage, Stuttgart I927.

Ferientage auf Sylt. Berlin 1928.

Moiken Peter Ohm. Roman. 2. Auflage, Stuttgart 1929.

Die letzten Sylter Riesen. Nach den Aufzeichnungen eines Zeitgenossen zusammengeatellt. Stuttgart 1930 .

Dammbau. Sylter Roman der Gegenwart. Stuttgart 1930.

Sylter Treue. Zwei Sagen von der Insel Sylt. Stuttgart 1932.

Bonhoff, Friedrich. Amrumer Grabsteine. Zeitschrift der Zentralstelle für Niedersächsische Familiengeschichte. Hamburg 1922.

Alte Grabsteine auf Föhr. Zeitschrift der Zentralstelle für Niedersächsische Familiengeachichte. Stade i. H. 1925 .

Borchling, C., und Muuß, R. Die Friesen. Breslau 1931.

Boysen, Jens. Beschreibung der Insel Sylt. Schleswig 1828.

Braren, Johann. Die vorgeschichtlichen Altertümer der Insel Föhr. Hamburg 1935.

Braun und Hogenberg. Civitates orbis terrarum etc. 1572-1618.

Brinner, Ludwig. Die deutsche Grönlandfahrt. Berlin 1913.

Brühl, Ludwig. Bernstein, das „Gold des Nordens". Meereskunde, Heft 166. Bd. XIV, Io. SIg. volkstümlicher Vorträge. Berlin.

Busch, Andreas. Die Entdeckung der letzten Spuren Rungholts. Jahrbuch des nordfriesischen Vereins Husum. 1923.

Taucht unser Land auch in der Gegenwart noch unter? Jahrbuch des nordfriesischen Vereins, Husum 1930.

Neue Gesichtspunkte zur Karthographie des mittelalterlichen Nordfriesland. Jahrbuch des Heimatbundes Nordfriesland. Husum 1936.

Neue Beobachtungen im Rungholt-Watt im Jahre 1935. Die Heimat, Heft 3. 1936.

Camerer, J. Fr. Sechs Schreiben von einigen Merkwürdigkeiten der holsteinischen Gegenden. Leipzig 1756.

Vermischte histor.-polit. Nachrichten in Briefen von einigen merkwürdigen Gegenden der Herzogtümer Schleswig und Holstein, ihrer natürlichen Geschichte und anderen seltenen Alterthümern. Flensburg und Leipzig I 758.

Christiansen, D. N. Die Blütenpflanzen und GefäBkryptogamen der Insel Föhr. Altona I925.

Christiansen, Julius. Zur Agrargeschichte der Insel Sylt. Mannheim 1923.

Christiansen, Willi. Die Vegetationsverhältnisse der Dünen auf Föhr. Leipzig I927.

Clement, Knut Jungbohn. Lebens- und Leidensgeschichte der Friesen. Kiel 1845.

Der Lappenkorb. Leipzig 1846.

Schleswig, das urheimische Land des nicht dänischen Volkes der Angeln und Friesen und Englands Mutterland, wie es war und ward. Hamburg 1862.

Danckwerth, Caspar. Newe Landesbeschreibung der zwey Hertzogthümer Schleswich und Holstein. 1652.

Dieren, J. W. van. Organogene Dünenbildung. Eine geomorphologische Analyse der Dünenlandschaft der West-Friesischen Insel Terschelling mit pflanzensoziologischen Methoden. Haag I934.

Dietz, Curt, und Heck, Herbert-Lothar. Geologische Karte von Deutschland I : 25000. Land Schleswig-Holstein. Erläuterungen zu den Blättern Sylt-Nord und Sylt-Süd. Aufgenommen von Curt Dietz. Landesanstalt für Angewandte Geologie, Kiel, 1952.

Dircksen, Rolf. Amrum. Ein erd-, natur- und volkskundlicher Wegweiser. Bielefeld 1936.

Die Insel der Vögel. Ein Buch von Austernfischern, Seeschwalben und Regenpfeifern. C. Bertelsmann Verlag. Gütersloh.

Engert, Rolf. Die Sage vom fliegenden Holländer. Meereskunde Band XV, 7. Berlin 1927.

Ernst, Otto. Zur Geschichte der Moore, Marschen und Wälder Nordwest-Deutschlands IV: Untersuchungen in Nordfriesland. Kiel 1934.

Eschels, Jens Jacob. Lebensbeschreibung eines Alten Seemannes von ihm selbst und zunächst für seine Familie geschrieben. Altona 1835 .

Dittmer, Ernst. Die Küstensenkung an der schleswig-holsteinischen Westküste. Forschungen und Fortschritte, Heft $17 / 18$. Berlin 1948 .

Mittelalterliche Verfehnung in Nordfriesland. Die Heimat 1950.

Das Eem des Treenetals. Schr. d. Naturw. Ver. f. Schl.-Holst., Band 25 „Gripp-Festschrift“ 195 I.

Die nacheiszeitliche Entwicklung der schleswig-holsteinischen Westküste. Meyniana. Band I. Schriften d. Geolog. Inst. d. Univ. Kiel. Neumünster 1952. 
Evers, Hans. Beiträge zur Microlepidopterenfauna von Sylt. 87 Arten der Sammlung von H. Koehn. Bombus Nr. 39, Hamburg, August 1947.

Ferrand, Dr. W. Hudig. Delfter Fayence. Berlin 1929.

Fischer, Otto. Die nordfriesischen Inseln vor und nach der Sturmflut vom 11. Oktober 1634. Berlin 1934. Weiteres siehe bei Müller, Friedrich.

Geerz, F. Geschichte der geographischen Vermessungen und der Landkarten Nordalbingiens vom Ende des 15. Jahrhunderts bis zum Jahre 1859. Berlin 1859.

Gering, Hugo. Beowulf nebst dem Finnsburg-Bruchstück. Heidelberg 1906.

Gripp, Karl, und Simon, Wilhelm Georg. Untersuchungen über den Aufbau und die Entstehung der Insel Sylt. I. Nord-Sylt.

Gripp, Karl, und Becker, Wilhelm. Untersuchungen über den Aufbau und die Entstehung der Insel Sylt. II. Mittel-Sylt. Westküste Archiv für Forschung, Technik und Verwaltung in Marsch und Wattenmeer. 2. Jahrgang. Doppelheft 2/3. Heide 1940.

Gripp, K., Stadermann, R., Schmidt, R., Jacob-Friesen, K. H. Werdendes Land am Meer. Berlin 1937.

Hāberlin, Carl. Beiträge zur Heimatkunde der Insel Fơhr. Wyk 1908.

Trauertrachten auf Föhr. Z. d. V. f. Volkskunde. Berlin. Heft 3, 1909.

Beitrag zur Geschichte von Wyk-Föhr. Die Halligwohnstätte. Das Brennmaterial der nordfriesischen Halligen. Aus: Friesen-Museum. Beiträge zur Heimatkunde von Föhr. Berlin Igrg.

Fohrer Urkunden nebst einem Faksimile der Burgurkunde von 1360. Wyk 1926.

Bunte Bilder aus der Föhrer Kulturgeschichte. Herausgegeben mit Dr. Roeloffs. Wyk r927.

Die Meeresheilkunde. Aus: Strahlentherapie. 31. Band, Heft 2. Berlin und Wien 1929.

Der Heilwert der Nordsee. Herausgegeben vom Verband Deutscher Nordseebäder. Berlin.

Seebäder. Bäder-Almanach, XV. Ausgabe. Berlin r 930.

Inselfriesische Volkstrachten vom 16. bis 18. Jahrhundert. Zeitschrift der Gesellschaft für Schleswig-Holsteinische Geschichte. Band 56 und 59. Neumünster 1926 und 1930.

Die nordfriesischen Salzsieder. Hamburg 1934.

Das Biiken in Nordfriesland. Aus: Die Heimat. Neumünster, Mai 1935.

Hăberlin, Carl, und Perlewitz, P. Klima-Atlas für die Meeresheilkunde an der deutschen Seeküste. Hamburg 1932.

Die Kur an den deutschen Seeküsten und ihre Wirkung nach den Ergebnissen der klimatophysiologischen Forschung dargestellt. Hamburg 1933.

Hagmeier, A., und Kändler, R. Neue Untersuchungen im nordfriesischen Wattenmeer und auf den fiskalischen Austernbänken. (Wissenschaftliche Meeresuntersuchungen, Abt. Helgoland, Band XVI. Abh. Nr. 6. 1927.)

Handelmann, Heinrich. Die amtlichen Ausgrabungen auf Sylt $1870-1872$. Kiel 1873 .

Die amtlichen Ausgrabungen auf Sylt 1873, 1875, 1877 und 1880. Kiel 1882.

Volks- und Kinder-Spiele aus Schleswig-Holstein. Kiel 1874.

Hansen, C. P. Die Insel Sylt in geschichtlicher und statistischer Hinsicht. Kiel 1845.

Friesische Sagen und Erzählungen. Altona 1858 .

Zur Geschichte der Halbinsel Hörnum auf Sylt. Jahrbücher für die Landeskunde der Herzogthümer Schleswig, Holstein und Lauenburg. Kiel 1859 .

Die nordfriesische Insel Sylt wie sie war und wie sie ist. Leipzig 1859.

Der Fremdenführer auf der Insel Sylt. Ein Wegweiser für Badende in Westerland. Mogeltondern 1859 .

Der Sylter Friese. Kiel 1860.

Ubbo der Friese. Schleswig 1864 .

Das Schleswig'sche Wattenmeer und die friesischen Inseln. Glogau 1865.

Der Badeort Westerland auf Sylt und dessen Bewohner. Garding 1868.

Die Friesen. Scenen aus dem Leben, den Kämpfen und Leiden der Friesen, besonders der Nordfriesen. 2. Ausgabe. Garding 1876 .

Chronik der friesischen Uthlande. 2. Auflage. Garding 1877.

Die Anfange des Schulwesens oder einer Schulchronik der Insel Sylt. Garding 1879.

Beiträge zu den Sagen, Sittenregeln, Rechten und der Geschichte der Nordfriesen. Deezbüll 1880 .

Hansen, Karl. Chronik von Pellworm. Husum 1938.

Hansen, Knud Melf. Chronikblätter der Nachkommen im Mánnesstamm des Broder Mumsen zu Bopslut im Nordstrande. Unter Mitwirkung von Adalbert Boysen. Band 1, 1908-1913. Band 2, 1923-1928. Detroit. Selbstverlag. 
Hansen, Reimer. Kurze Schleswig-Holsteinische Landesgeschichte. 2. Auflage. Flensburg 1924

Haupt, Richard. Die Bau- und Kunstdenkmäler der Provinz Schleswig-Holstein. Band I-III. Kiel 1887 und 1889 .

Heimat, Die. Monatsschrift für schleswig-holsteinische Heimatforschung und Volkstumspflege. Kiel. Heimreich, M. Antoni. Ernewerte Nordfresische Chronick. Schleswig 1666. 2. Auflage 1668. Faksimile-Neudruck, München 1926.

Hepp, C. Praktischer Wegweiser auf der Insel Sylt. 4. Auflage. Tondern und Westerland 1887.

Herrmann, Paul. Dänische Heldensagen nach Saxo Grammaticus. Jena 1925.

$\mathrm{HeB}, \mathrm{W}$. Erinnerungen an Sylt. Hannover 1876 .

Hinrichs, W. Nordsee. Deiche, Küstenschutz und Landgewinnung. Husum 1931.

Heydemann, F. Schmetterlingsfauna der Insel Amrum. Aus den Schriften des Naturwissenschaftlichen Vereins für Schleswig-Holstein. Band XX, Heft 2. 1934.

Hinz, H. Zur Herkunft der Nordfriesen. Jahrbuch des Nordfriesischen Vereins für Heimatkunde und Heimatliebe. Band 29, Jahrgang 1952/53.

His, Rudolf. Der Totenglaube in der Geschichte des germanischen Strafrechts. Münster I929.

Hoffmann, Anna. Die Landestrachten von Nordfriesland. Westholsteinische Verlagsanstalt Boyens \& Co., Heide in Holstein, 1940.

Hübbe, Andreas. Söl'ring Dechtings en Leedjis ütdön fuar di Söl'ring Jungen. Hamburg 1911.

Illies, Henning. Die Schrägschichtung in fluviatilen und litoralen Sedimenten, ihre Ursachen, Messung und Auswertung. Aus den Mittlg. a. d. Geolog. Staatsinstitut in Hamburg. Heft 19/1949, S. 89-109.

Jahrbuch des Heimatbundes Nordfriesland. Husum. (Früher: Jahrbuch bzw. Mitteilungen des Nordfriesischen Vereins für Heimatkunde und Heimatliebe. Husum. Heft 1. 1903/04.)

Jahrbücher für die Landeskunde der Herzogthümer Schleswig, Holstein und Lauenburg. Band I_-X. 1858-1869. Kiel.

Jannen, Johannes E. Aus den Tagen unserer Väter. Wyk 1932.

Jensen, Christian. Vom Dünenstrand der Nordsee und vom Wattenmeer. Schleswig 1900. Bestrebungen zur Erhaltung des Nordfriesischen Volkstums im 19. Jahrhundert. Schleswig 1909. Selbstverlag.

Inseln in der Sage. Schleswig 1910. Selbstverlag.

Aus Sturm und Not. Erzählungen und Skizzen vom Nordseestrand. Westerland 1913.

Die nordfriesische Inselwelt. 2. Auflage. Braunschweig-Hamburg 1925.

Friesische und Schweizer Wandersagen. Abhandlungen zur Meeresheilkunde und Heimatkunde der Insel Föhr und Nordfrieslands. Leipzig 1927.

Die nordfriesischen Inseln Sylt, Föhr, Amrum, Helgoland und die Halligen vormals und jetzt. 2. Auflage. Lübeck 1927.

Vom Tanz der Inselfriesen. Schleswig 1930.

Jessen, Otto. Morphologische Beobachtungen an den Dünen von Amrum, Sylt und Röm. Mitt. d. Geogr. Ges. in München. Band 9. 1914 .

Jessen, Werner. Die postdiluviale Entwicklung Amrums und seine subfossilen und rezenten Muschelpflaster. (Unter Berücksichtigung der gleichen Vorgänge auf den Inseln Sylt und Föhr.) Jahrbuch der Preußischen Geologischen Landesanstalt für 1932. Band 53. Berlin 1932.

Jessen, Wilhelm. Rantum auf Sylt. Teil I und 2. Westerland Ig24 und 1925. Sylter Sagen. Nach den Schriften des Heimatforschers C. P. Hansen. Westerland 1925.

Jessen, Wilhelm. Uwe Jens Lornsens Vorfahren und ihre Welt. Zeitschrift d. Ges. f. SchleswigHolsteinische Geschichte. Band 66. Sonderdruck, Karl Wachholtz Verlag, Neumünster in Holstein.

Johansen, Chr. Halligenbuch. Eine untergehende Inselwelt. 2. Auflage. Schleswig 1889.

Jorgensen, Peter. Über die Herkunft der Nordfriesen. Kobenhavn 1946.

Kersten, Karl. Die vorgeschichtliche Landesaufnahme von Sylt. Ausgrabungen auf Sylt. Nachrichtenblatt für Deutsche Vorzeit. Leipzig 1942. Heft 3/4.

Kersten, Karl und Peter La Baume unter Mitwirkung von Herbert Jankuhn, Ernst Dittmer und Alfred Rust. Vorgeschichte der nordfriesischen Inseln. Neumünster 1958.

Kielholt, Hans. Silter Antiquitäten. (Zeit etwa 1435.) Herausgegeben von N. Falck in „M. Anton Heimreichs, norfresische Chronik". II. Theil. III. Auflage. Tondern I819.

Koehn, Henry. Sylt. Ein Führer durch die Inselwelt. 2. berichtigte und verbesserte Auflage, Hamburg 1960 .

Kohl, J. G. Die Marschen und Inseln der Herzogthümer Schleswig und Holstein. 3 Bände. Dresden XVI und Leipzig 1846 . 
Die erste deutsche Entdeckungsreise zum Nordpol. Bremisches Jahrbuch, Band 5. Bremen 1870.

Kolumbe, Erich. Das Naturgebiet Listerland auf Sylt. Nordelbingen. Band 7. Flensburg 1928.

Ein Beitrag zur Kenntnis der Entwicklungsgeschichte des Königshafens bei List auf Sylt. Wissenschaftliche Meeresuntersuchungen. Abtlg. Kiel. XXI. Band. Kiel 1932.

Sylt. Ein Insellesebuch. Unterrichtliche Merkblätter für die Hand des Lehrers. Heft 5. Hamburg 1951.

Konietzko, J. Die volkstümliche Kultur der Halligbewohner. Niederdeutsche Zeitschrift für Volkskunde. Jahrgang 8, Heft 1 und 3/4, Bremen 1930. Jahrgang 9, Heft 3/4, Bremen 193 I.

Krohn, Hugo. Bilderahnentafel der Geschwister Matzen, Wenningstedt.

Die Sippe der Nordmark. Heft 2. 1937.

Uwe Jens Lornsens Vorfahren. Die Sippe der Nordmark. Folge 2. 1938.

Die Bev8lkerung der Insel Sylt. Inaugural-Dissertation. Kiel 1949.

Krüger, Edgar. Die Hummeln und Schmarotzerhummeln von Sylt und dem benachbarten Festland. Schriften d. naturw. Ver. f. Schleswig-Holstein: Band XXIII, Heft I. 1939.

La Baume, Peter. Die Wikingerzeit auf den Nordfriesischen Inseln. Jahrbuch des Nordfriesischen Vereins für Heimatkunde und Heimatliebe. Band 29, Jahrgang 1952/53.

Lamprecht, Heinz-Otto. Brandung und Uferveränderungen an der Westküste von Sylt. Mitteilungen der Hannoverschen Versuchsanstalt für Grundbau und Wasserbau. Franzius-Institut der Technischen Hochschule Hannover. Heft 8, I955.

Laur, Wolfgang. Die Ortsnamen Nordfrieslands. Jahrbuch des Nordfriesischen Vereins. Band 29, Jahrgang 1952/53.

Lehmann, Otto. Das Bauernhaus in Schleswig-Holstein. Altona 1927. Spiele und Spielzeug in Schleswig-Holstein. Jahrbuch für historische Volkskunde. III. und IV. Band. Berlin 1934.

Leistner, Walter. Das Wattenmeer- und Küstenklima Nordfrieslands und sein EinfluB auf den menschlichen Organismus. Reichsamt für Wetterdienst. Wiss. Abhdlg., Band 5. Berlin 1938.

Lornsen, Uwe Jens. Die Unions-Verfassung Dänemarks und Schleswigholsteins. Herausgegeben von Georg Beseler. Jena 184 I.

Lübbing, Hermann. Friesische Sagen von Texel bis Sylt. Jena 1928.

Mager, Friedrich. Der Abbruch der Insel Sylt durch die Nordsee. Breslau I927.

Martens, Friedrich. Fridrich Martens vom Hamburg Spitzbergische oder Groenlandische Reise Beschreibung gethan im Jahr 1671 . Hamburg 1675 .

Meiborg, R. Das Bauernhaus im Herzogtum Schleswig. Schleswig 1896.

Meyer, Gustav Fr. Schleswig-Holsteiner Sagen. Jena 1929.

Meyn, L. Geognostische Beschreibung der Insel Sylt und ihrer Umgebung. 1876.

Möller, Boy P. Söl'ring Leesbok. Altona 1909. Solring Uurterbok. Hamburg 1916.

Möller, Theodor. Die Welt der Halligen. Kiel 1924.

Mohr, Erna. Die Landsäugetiere der schleswig-holsteinischen Nordsee-Inseln. Schriften des Naturwissenschaftlichen Vereins für Schleswig-Holstein. Band XIX, Heft 1. Kiel und Leipzig Igrg.

Müllenhoff, Karl. Sagen, Märchen und Lieder der Herzogtümer Schleswig, Holstein und Lauenburg. Neue Ausgabe. Schleswig 1921 .

Müller, Friedrich. Das Wasserwesen an der Schleswig-Holsteinischen Nordseeküste. Die Halligen, Band I und II. Berlin 1917. Alt-Nordstrand, Berlin 1936; Nordstrand, Berlin 1936; Pellworm, Berlin 1936; Amrum, Berlin 1937; Föhr, Berlin 1937; Sylt, Berlin 1938; Allgemeines. Berlin 1938; bearbeitet und ergänzt von Dr. O. Fischer.

Mungard, Nann. For Sölring Spraak en Wiis. Eine Sammlung von Sylter Wörtern, wie sie zu Anfang des 20. Jahrhunderts auf Sylt gesprochen und vordem gebraucht worden sind, Westerland 1909 .

Muuß, Rudolf. Rungholt. Ruinen unter der Friesenhallig. Lübeck.

Nerong, O. C. Föhr früher und jetzt. Selbstverlag 1885 .

Chronik der Familie Flor. Selbstverlag 1887.

Das Dorf Wrixum. Historisch und topographisch beschrieben. 1898 .

Die Insel Föhr. Selbstverlag 1903.

Die Kirchhöfe Föhrs. 3. Auflage. Selbstverlag rgog.

Niemeyer, Wilhelm. Oluf Braren, der Maler von Föhr. Berlin 1920.

Nöbbe, Erwin. Münzfund von Westerland auf Sylt. Mittlg. d. Anthropolog. Vereins in SchleswigHolstein. Heft 19, Kiel I9I r.

Ein Silberschmuck der Wikingerzeit von List auf Sylt. Nachrichtenblatt für Deutsche Vorzeit. Leipzig 1940. Heft $4 / 5$.

XVII 
Oesau, Wanda. Schleswig-Holsteins Grönlandfahrt auf Walfischfang und Robbenschlag vom 17. bis 19. Jahrhundert. Glückstadt-Hamburg-New York 1937.

Die deutsche Südseefischerei auf Wale im 19. Jahrhundert. Glückstadt-Hamburg-New York 1939.

Olshausen, Otto. Amrum. Bericht über Hügelgräber auf der Insel nebst einem Anhange ubber die Dünen. Berlin 1920.

Ottsen. Die Nordseeinsel Sylt. Erdkundliche und geschichtliche Betrachtungen. Westerland rgro Pappenheim, Max. Die Siebenhardenbeliebung vom 17. Juni 1426. Flensburg 1926.

Pauls, Volquart. Uwe Jens Lornsen's Briefe an Franz Hermann Hegewisch. Schleswig rg25. Die Eiderstedter Freiberge. Abhandlung zur Meeresheilkunde und Heimatkunde der Insel Fohr und Nordfrieslands. Leipzig 1927.

Peters, L. C. Das föhringische Haus. Mitteilungen des nordfriesischen Vereins für Heimatkunde und Heimatliebe 1911/12.

Nordfriesland. Heimatbuch für die Kreise Husum und Südtondern. Herausgegeben von L. C. Peters. Husum 1929.

Zwischen West- und Nord-Germanien. Beitrăge zur Heimatkunde der nordfriesischen Uthlande und der benachbarten Geestharden für Schule und Haus. Husum 1932.

Petersen, Egon. Wyk. Ein Überblick über seine Geschichte. Wyk I93o.

Petreus, Johannes. Schriften über Nordstrand. Herausgegeben von Reimer Hansen. Kiel 1910. Philippsen, H. Sagen und Sagenhaftes der Insel Föhr. 2. Auflage. Garding 1928.

Kultur- und Naturbilder von Forhr. 3. Auflage. Garding 1928.

Pielenz, Otto Karl. Neue Forschungsergebnisse über die alt- und mittelsteinzeitliche Kulturentwicklung in Schleswig-Holstein. Mannus, Z. f. Deutsche Vorgeschichte. Leipzig 1937. Band 29. Heft 4.

Ein Siedlungsplatz des Magdalónien auf Sylt. Veröffentlichungen der Sammlung Otto Karl Pielenz-Eidelstedt. Nr. 2. Hamburg 1940.

Die Entstehung der Hacke aus dem Lochstab des Zweigeschlechterkultes. Veroffentlichungen der Sammlung Otto Karl Pielenz-Eidelstedt. Nr. 5. Hamburg 1946.

Die altpaläolıthische Fundstelle Emmerleff Kliff in Nordschleswig. Veröfftlg. d. Slg. O. K. Pielenz, Hamburg 1955.

Faustkeile aus Felsgestein aus dem Alt-Pleistozän vom Roten Kliff auf Sylt. Veröfftlg. d. Slg. O. K. Pielenz, Hamburg 1956.

Das Altpaläolithikum in Dänemark. Forschungen und Fortschritte, Bd. 34, 1960.

Die Bedeutung der späteiszeitlichen Siedlungsfunde auf Sylt für die Entstehung der mittelsteinzeitlichen Kulturen Nordeuropas. Forschungen und Fortschritte, Bd. 34, 1960.

Plett, Emil. Zur Rechtsgeschichte des Spätlandes auf Osterlandföbr und der am Spätland bestehenden Interessentschaften. Leipzig 1931 .

Plinius, Cajus Secundus. Naturgeschichte. Úbersetzt von C. F. L. Strack. Bremen 1854 .

Pontoppidan, Erich. Danske Atlas. Kopenhagen 1763-1 781 .

Rasmussen, Knud. Heldenbuch der Arktis. Entdeckungsreisen zum Nord- und Südpol. Leipzig 1933.

Reimers, Heinrich. Friesische Papsturkunden aus dem Vatikanischen Archive zu Rom. Leeuwarden I908.

Das Papsttum und die freien Friesen. Aus: „De Vrye Fries“. 28. Teil. Leeuwarden 1928.

Reinke, J. Die Entwicklungsgeschichte der Dünen an der Westküste Schleswigs. Sitzungsbericht d. Kgl. Akad. d. Wissensch. Berlin rgo3.

Botanisch-geologische Streifzüge an der Küste des Herzogtums Schleswig. Wissenschaftliche Meeresuntersuchungen. Abt. Kiel. Band VIII, Ergänzungsheft 1903.

Retzlaff, Hans. Deutsche Bauerntrachten. Berlin 1934.

Riemann, Else. Nordfriesland in der erzählenden Dichtung. Probefahrten, Band 16. Leipzig 1910.

Rieter, J. Danske nationale Klaededragter. Kopenhagen 1805,1806 und 1811 .

Renger-Patzsch, A. Die Halligen. Geleitwort von Johann Johannsen. Berlin 1927.

Rust, Alfred. Das altsteinzeitliche Rentierjägerlager Meiendorf. Neumünster 1937.

Artefakte aus der Zeit des Homo Heidelbergensis in Süd- und Norddeutschland. Bonn 1956.

Sach, August. Das Herzogtum Schleswig in seiner ethnographischen und nationalen Entwicklung. Halle 1896.

Sauermann, Ernst. Die mittelalterlichen Taufsteine der Provinz Schleswig-Holstein. Flensburg 1904. Herausgeber von „Die Kunstdenkmäler der Provinz Schleswig-Holstein“. Die Kunstdenkmäler des Kreises Husum. Bearbeitet von Heinrich Brauer, Wolfgang Scheffler, Hans Weber. Berlin 1939. Die Kunstdenkmäler des Kreises Südtondern. Bearbeitet von Heinrich Brauer, Wolfgang Scheffler, Hans Weber. Berlin 1939. 
Sax, Peter. Ein Beschreibung der Insul und landes Nordtstrandt. 1637. Herausgegeben von Emil Bruhn. Mittlg. d. nordfries. Vereins für Heimatkunde und Heimatliebe. Heft 6. rgog/10.

Saxo Grammaticus. Historia Danica. Herausgegeben von P. E. Müller. Kopenhagen I839-I858.

Schade. Wasserbauamt Husum. Denkschrift über die bisherigen Erfahrungen mit den Buhnen am Weststrande von Sylt. Husum 1936.

Scheel, Otto. Die Frühgeschichte bis r 10o. Geschichte Schleswig-Holsteins. Band 2, 2. Hälfte, Lieferung 1. Neumünster 1938 .

Die Heimat der Angeln. Aus: Festgabe zur ersten Jahrestagung des Instituts für Volks- und Landesforschung an der Universität Kiel. Neurnünster I939.

Scheltema, F. Adama von. Die Altnordische Kunst. 2. Auflage. Leipzig 1923.

Die Kunst unserer Vorzeit. Leipzig 1936.

Schmidt, Hermann. Die Windmühlen der Insel Sylt. Nach alten Chroniken, Urkunden und mündlichen Überlieferungen für die Jugend der Insel bearbeitet. Husum 1937.

Zur Heimatkunde der Insel Sylt. Heft 1. 1. Zur ursprünglichen Lage des Ortes Braderup auf Sylt. 2. Úber Deicharbeiten der Holländer auf Sylt. Husum 1938. - Zur Chronik. des Ortes Tinnum. Sylter Nachrichten. Westerland 1937-39.

Schmidt-Petersen, J. Wörterbuch und Sprachlehre der nordfriesischen Sprache nach der Mundart von Föhr und Amrum. Husum ig12.

Die Orts- und Flurnamen Nordfrieslands. Herausgegeben vom Nordfriesischen Verein für Heimatkunde. Husum 1925.

Schott, C. Die schleswig-holsteinische Westküste. Probleme der Küstensenkung. Mitteilung des Geogr. Inst. d. Univ. Kiel. r951.

Schütte, H. Krustenbewegungen an der deutschen Nordseeküste. Aus der Heimat, Heft I I. Stuttgart 1927.

Schulz, Bruno. Die deutsche Nordsee, ihre Küsten und Inseln. Monographien zur Erdkunde. Bielefeld und Leipzig 1928.

Schwantes, Gustav. Die Vorgeschichte Schleswig-Holsteins. (Stein- und Bronzezeit.) Neumünster 1939.

Siebs, Theodor. Zur Geschichte der englisch-friesischen Sprache, I. Halle 1889. Geschichte der friesischen Sprache. Grundriß der germanischen Philologie I.

Spanuth, Jürgen, Pastor. Stollberg - ein altes friesisches Zentralheiligtum. Jahrbuch des Heimatbundes Nordfriesland. Band 25. Jahrgang 1938.

Stahl, Wilhelm. Volkstänze von den nordfriesischen Inseln. Kassel.

Stierling, Hubert. Der Silberschmuck der Nordseeküste hauptsächlich in Schleswig-Holstein. Neumünster 1935 .

Stolley, E. Geologische Mitteilungen von der Insel Sylt, I-III. Archiv für Anthropologie und Geologie Schleswig-Holsteins, Band III und IV. I900/or.

Tacitus, C. Germania. Herausgegeben aus dem Lateinischen von F. W. Tönnies. Berlin 1816.

Tedsen, Julius. Der Lautbestand der föhringischen Mundart. Halle 1906. Zeitschrift für deutsche Philol., 36 und 39 .

Erlebnisse nordfriesischer Seeleute. Langensalza 1937.

Thiergart, Fr. Zur Altersbestimmung eines Saprohumoliths am Roten Kliff auf Sylt zwischen Wenningstedt und Kampen (Buhne 31). In Mikropaläobotanische Mitteilungen, I.-3. Jb., Reichsstelle für Bodenforschung für 194I, Band 62. Berlin 1942.

Timm, R. Moose auf der Insel Föhr. Ein Beitrag zur Naturgeschichte dieses Eilandes. Wyk 1926.

Traeger, Eugen. Das Erdbuch der Hallig Hooge. Zeitschrift der Gesellschaft für SchleswigHolsteinische Geschichte. 31. Bariú Kiel Igor.

Ulrich, Käthe. Die Morphologie des Roten Kliffs auf Sylt. Archiv der Deutschen Seewarte. 56. Band, Nr. I. Hamburg 1936.

Varges, Helene. Flutkante und Inselflora. Neumünster 1936.

Vogel, Walther. Zur nord- und westeuropäischen Seeschiffahrt im frühen Mittelalter. Hansische Geschichtsblätter, XIII. 1907.

Voigt, Ehrhard. Die Anwendung der Lackfilm-Methode bei der Bergung geologischer und bodenkundlicher Profile. Aus den Mitteilungen des Geologischen Staatsinstituts in Hamburg, Heft 19, S. I I 1-1 29 . 
Warnecke, Georg. Eiszeit und Nacheiszeit in ihrem Einfluß auf die Zusammensetzung der Schmetterlingsfauna Schleswig-Holsteins. Nordelbingen. Flensburg 1928.

Die Großschmetterlinge der nordfriesischen Insel Sylt. Geographisch-historische, ökologische und genetische Probleme der Fauna Sylts. (Literaturübersicht beigegeben.) Stuttgart 1937.

Für die nordfriesischen Inseln neue Großschmetterlinge. Bombus, Nr. 25. Hamburg, April I 943 .

Jungzeitliche Strandformen unter den Schmetterlingen der deutschen Nordseeküsten. Bombus, Nr. 37, Hamburg, Juni 1947 , und Nr. $3^{8}$, Juli 1947.

Einige Bemerkungen zur Frage der Variabilität bei den Schmetterlingen der friesischen Inseln. Verhandl. d. Ver. f. naturw. Heimatforschung zu Hamburg, Band 29, I947.

Weber, Karl. Zur Rechtsgeschichte der Wiesengemeinschaften der Hallig Hooge. Leipzig $193 \mathbf{I}$.

Weckmann-Wittenburg, P. F. Norderoog. Ein deutsches Vogelparadies. Natururkunden von den Halligen und vom Wattenmeer. Berlin $193 \mathrm{I}$.

Wegner, Theodor. Vorläufige Mitteilungen über Studien im nordfriesischen Wattgebiet. Zentralblatt f. Min. usw., Abt. B, Nr. 5, Jahrg. 1931.

Weigelt, G. Die nordfriesischen Inseln vormals und jetzt. Eine Skizze des Landes und seiner Bewohner. 2. Auflage. Hamburg 1873.

Westphalen, E. J. de. Rerum Germanicorum etc. Monumenta inedita. Leipzig 1739.

Wibel, F. Der Gangbau des Denghoogs bei Wenningstedt auf Sylt. Jahrbücher für die Landeskunde der Herzogthümer Schleswig, Holstein und Lauenburg. Band X. Kiel 1869.

Wirtz, Daniel. Die Fauna des Sylter Crag und ihre Stellung im Neogen der Nordsee. Mitteilungen aus dem Geologischen Staatsinstitut in Hamburg. Heft 19, S. 57-76. Hamburg 1949.

Wiser, Friedrich Ritter von. Die Besetzung der nordfriesischen Inseln im Juli 1864. (Verfaßt im Jahre I864.) „Danzer's Armee-Zeitung" und Sonderdruck, Wien I914.

Woebcken, Carl. Deiche und Sturmfluten an der deutschen Nordseeküste. Bremen 1924.

Wohlenberg, Erich. Die Grüne Insel in der Eidermündung. Eine entwicklungsphysiologische Untersuchung. Archiv der Deutschen Seewarte, 5o. Band, Nr. 2. Hamburg 1931.

Ruinen im Wattenmeer. Natur und Museum. 1932.

Beobachtungen über das Seegras, Zostera marina, und seine Erkrankung im nordfriesischen Wattenmeer. Nordelbingen. Band I1. 1935.

Die Lebensgemeinschaften im Königshafen von Sylt. Helgoländer Wissenschaftliche Meeresuntersuchungen. Band I, I. 1937.

Biologische Kulturmaßnahmen mit dem Queller (Salicornia herbacea) zur Landgewinnung im Wattenmeer. Westküste. Band I, 2. 1938.

Wolf, Wilhelm. Erdgeschichte und Bodenaufbau Schleswig-Holsteins unter Berücksichtigung des nordhannoverschen Nachbargebietes. 3. Auflage. Hamburg 1949.

Die Entstehung der Insel Sylt. 4, völlig neu bearbeitete Auflage. Hamburg 1938.

Zimmermann, Ernst. Chinesisches Porzellan. Leipzig 1923.

Zorgdrager, C. G. Groenlandsche Visschery. Amsterdam 1728. 